\title{
The SIRveNIB and SARAH trials, radioembolization vs. sorafenib in advanced HCC patients: reasons for a failure, and perspectives for the future
}

\author{
Carlo Sposito $^{1}$, Vincenzo Mazzaferro ${ }^{1,2}$ \\ ${ }^{1}$ Department of Gastrointestinal Surgery and Liver Transplantation, Fondazione IRCCS Istituto Nazionale Tumori di Milano, Milan, Italy; \\ ${ }^{2}$ University of Milan, Milan, Italy \\ Correspondence to: Carlo Sposito. Department of Gastrointestinal Surgery and Liver Transplantation, Fondazione IRCCS Istituto Nazionale Tumori \\ di Milano, Milan, Italy. Email: carlo.sposito@istitutotumori.mi.it. \\ Comment on: Chow PKH, Gandhi M, Tan SB, et al. SIRveNIB: Selective Internal Radiation Therapy Versus Sorafenib in Asia-Pacific Patients With \\ Hepatocellular Carcinoma. J Clin Oncol 2018;36:1913-21.
}

Submitted Oct 08, 2018. Accepted for publication Oct 17, 2018.

doi: 10.21037/hbsn.2018.10.06

View this article at: http://dx.doi.org/10.21037/hbsn.2018.10.06

Liver cancer is the fifth most common cancer and the second most frequent cause of cancer-related death globally. Hepatocellular carcinoma (HCC) represents nearly $90 \%$ of primary liver cancers, and constitutes a major health problem worldwide (1). When diagnosed at an early stage of the disease, HCC may benefit from potentially curative treatments such as liver resection, liver transplantation or local ablation. Despite effectiveness of treatment in early and very early stages, most patients are diagnosed or progress to an intermediate or advanced stage, in which treatment options are limited and the prognosis is poor (2).

Up to now the only systemic therapy in first line that has shown to confer survival advantage in patients with advanced HCC is sorafenib, an oral multikinase inhibitor $(3,4)$. Sorafenib has become the reference treatment for HCC patients in the advanced stage of the disease, and in those patients at the intermediate stage refractory or not amenable to locoregional treatments (5). However, the survival advantage provided by sorafenib is relatively small (in the range of 2-3 months), radiological responses are rare and discontinuation and dose reduction of the drug are frequently necessary because of adverse events such as diarrhoea, fatigue, and hand-foot skin reaction $(3,4)$.

Radioembolization (RE) with yttrium-90 (90Y) microspheres is a form of brachytherapy delivered via the hepatic artery, that allows targeted delivery of high-dose radiation to liver tumours. Several retrospective and large cohort studies showed an acceptable safety profile of RE, and good results in terms of local control of the disease and long-term survival in patients with unresectable HCC limited to the liver in the intermediate and advanced stages (6-8). No randomized clinical trials (RCTs) comparing the efficacy of RE with respect to sorafenib had been published until recently, and as a consequence RE is still not recommended in international guidelines.

SIRveNIB (selective internal radiation therapy $v s$. sorafenib) is a recently published open-label phase III trial that compared $90 \mathrm{Y}$-resin microspheres RE with sorafenib $800 \mathrm{mg} / \mathrm{d}$ in patients with locally advanced HCC, in a two-tailed study designed for superiority/detriment; the primary end point was overall survival (OS). The trial randomised 360 patients (182 to RE, 178 to sorafenib) across 11 countries in the Asia-Pacific region. The study failed to meet the primary endpoint: in fact, median OS in the intention-to-treat population was 8.8 and 10.0 months with $\mathrm{RE}$ and sorafenib, respectively (hazard ratio, 1.1; 95\% CI: 0.9-1.4; $\mathrm{P}=0.36$ ). Similarly, no differences in OS were demonstrated in the treated population (11.3 and 10.4 months in the RE and sorafenib arms, respectively, $\mathrm{P}=0.27$ ), nor in subgroup analyses. Tumor response rates (TRR) were significantly higher in the RE arm, however disease control rates were similar and no differences in progression-free survival were observed. Fewer patients in the RE group (20.8\%) than in the sorafenib group (35.2\%) had serious AEs, and mean duration of side effects was shorter in the RE arm. These results, however, did not 
translate into significant differences in patients' quality of life since the EQ-5D index between the RE and sorafenib groups was similar throughout the study (9).

The SARAH trial (SorAfenib versus Radioembolization in Advanced Hepatocellular carcinoma) enrolled 467 patients (237 RE, 222 sorafenib) from 25 centers in France. Similiarly to SIRveNIB, it was a phase III trial designed for superiority that compared 90Y-resin microspheres RE with sorafenib $800 \mathrm{mg} / \mathrm{d}$ in patients with locally advanced HCC. Also this Western-based trial failed to meet the primary endpoint since median OS was 8.0 months in the RE group vs. 9.9 months in the sorafenib group (hazard ratio, 1.15; 95\% CI: 0.94-1.41; $\mathrm{P}=0.18)(10)$.

As stated elsewhere, failure of rejecting the null hypothesis in a superiority trial should be distinguished from the concept used in a non-inferiority trial, in which a non-inferiority margin is set a priori and generally larger sample sizes are needed (11). Consequently, neither the results of SIRveNIB nor the results of SARAH trials could be instrumental to claim for an equivalency between RE and sorafenib. Thus, how should the results of these two recent RTCs be interpreted in the light of the previously published studies and clinical experience, that showed a promising efficacy of RE even if at a lower level of evidence? Should $\mathrm{RE}$ be abandoned as a treatment for HCC or should it be pursued in some specific subsets of patients? To answer these questions, we think that the difficulties and potential biases undermining trials that compare an interventional procedure (RE) to a drug (sorafenib) should be carefully analysed.

Radioembolization is a sophisticated technical procedure that requires high skills and a multidisciplinary management involving several medical and non-medical figures, after a considerable learning curve. The expertise in managing such treatment might have been highly heterogeneous in both the SIRveNIB trial (which was conducted at 11 centers, among which only 5 had facilities to perform RE) and the SARAH trial (which was conducted at 25 centers). Most of the cohort studies reporting promising results of $\mathrm{RE}$ were conducted on single center series, from highly experienced centers (12): the relatively poor results of RE in both RCTs might be partially explained by a diluting effect of the multicentricity of the design, with some contributing centres having little experience of administering RE. In such view, a subgroup analysis evaluating the results according to center expertise or number of procedures performed might have helped to clarify this issue.

Considering that RE is a form of radiotherapy, dosimetric considerations should be further elucidated. In the two RCTs, the planned activity and dose $90 \mathrm{Y}$ treatment were based on the body surface area: no endpoints regarding tumour-absorbed dose and liver-absorbed dose were planned, while a clear tumour dose-response relationship with glass microspheres had been demonstrated in several studies (13).

In both the SIRveNIB and the SARAH trial, patients waited after randomization a median time of 21 and 29 days to receive RE, and a median time of 3 and 7 days to receive sorafenib respectively. This discrepancy of nearly 3 weeks of "no treatment" between the two treatment arms was not accounted in the ITT nor in the per-protocol analyses, which calculate OS from the time of randomization. In the view of post-treatment survival, this is a lead time likely to impact on patients with a short life expectancy. Moreover, in both trials there were more patients who did not receive the assigned treatment in the RE than in the sorafenib group. This discrepancy was partially explained by the fact that some patients had worsening of the disease during the preRE work-up that contraindicated SIRT, and clearly acted as a negative selection bias.

Finally, the appropriate population in which a benefit of $\mathrm{RE}$ over sorafenib could be shown still needs to be defined. The most promising results of RE had been observed in cohort studies focused only on a specific population, such as patients with HCC and tumoral portal vein thrombosis (PVT) $(8,12)$. A local treatment such as RE, as well as surgical resection, demonstrated to be particularly effective in those patients with PVT limited to primary or secondary order branches of the portal vein, and less or noneffective in patients with occlusion of the main portal trunk $(12,14,15)$. In both the SIRveNIB and SARAH trials, subgroup analyses did not demonstrate a significant survival benefit in patients with PVT undergoing RE. Apart from being underpowered by definition, subgroup analyses in both studies were not designed to target survival differences according to PVT extension. To better evaluate the effectiveness of first-line $\mathrm{RE}$ with respect to sorafenib in the specific subset of HCC patients with PVT, only one RCT (Yes-p, NCT01887717) was run but it was prematurely terminated because of poor accrual. In retrospective the Yes-p, would it be supported in its premise by both interventional radiology and oncology communities, remains the only study with intact hypothesis of superiority of RE over sorafenib.

In conclusion, the SIRveNIB and the SARAH trial failed to prove a superiority of $\mathrm{RE}$ over sorafenib in patients with advanced HCC. However, these trials were pivotal since they were the first RCTs confirming the safety and efficacy 
of RE in patients with locally advanced HCCs. Both studies suggested that RE might be better tolerated than sorafenib in that patients, and these results are in line with what is felt by most clinicians dealing with RE. A clear superiority of $\mathrm{RE}$ with respect to sorafenib in inducing tumor response was confirmed: the reasons why such a result did not translate into a benefit in OS nor in PFS should be probably researched in a better and more restrictive patients selection for the procedure. In particular, until new trials will be designed, we think that the results of large cohort studies demonstrating that RE provides a significant survival benefit in patients with HCC and PVT (12) should not be disregarded because of the present two negative RCTs, that included a broader spectrum of intermediate and advanced HCC patients.

\section{Acknowledgements}

None.

\section{Footnote}

Conflicts of Interest: C Sposito and V Mazzaferro received honoraria from BTG for training courses with educational purposes on radioembolization.

\section{References}

1. Akinyemiju T, Abera S, Ahmed M, et al. The Burden of Primary Liver Cancer and Underlying Etiologies From 1990 to 2015 at the Global, Regional, and National Level: Results From the Global Burden of Disease Study 2015. JAMA Oncol 2017;3:1683-91.

2. European Association for the Study of the Liver. EASL Clinical Practice Guidelines: Management of hepatocellular carcinoma. J Hepatol 2018;69:182-236.

3. Llovet JM, Ricci S, Mazzaferro V, et al. Sorafenib in advanced hepatocellular carcinoma. $\mathrm{N}$ Engl J Med 2008;359:378-90.

4. Cheng AL, Kang YK, Chen Z, et al. Efficacy and safety of sorafenib in patients in the Asia-Pacific region with advanced hepatocellular carcinoma: a phase III randomised, double-blind, placebo-controlled trial. Lancet Oncol 2009;10:25-34.

5. Bruix J, Raoul JL, Sherman M, et al. Efficacy and safety of sorafenib in patients with advanced hepatocellular carcinoma: subanalyses of a phase III trial. J Hepatol 2012;57:821-9.

6. Sangro B, Carpanese L, Cianni R, et al. Survival after yttrium-90 resin microsphere radioembolization of hepatocellular carcinoma across Barcelona clinic liver cancer stages: a European evaluation. Hepatology 2011;54:868-78.

7. Salem R, Lewandowski RJ, Mulcahy MF, et al. Radioembolization for hepatocellular carcinoma using Yttrium-90 microspheres: a comprehensive report of longterm outcomes. Gastroenterology 2010;138:52-64.

8. Mazzaferro V, Sposito C, Bhoori S, et al. Yttrium-90 radioembolization for intermediate-advanced hepatocellular carcinoma: a phase 2 study. Hepatology 2013;57:1826-37.

9. Chow PKH, Gandhi M, Tan SB, et al. SIRveNIB: Selective Internal Radiation Therapy Versus Sorafenib in Asia-Pacific Patients With Hepatocellular Carcinoma. J Clin Oncol 2018;36:1913-21.

10. Vilgrain V, Pereira H, Assenat E, et al. Efficacy and safety of selective internal radiotherapy with yttrium-90 resin microspheres compared with sorafenib in locally advanced and inoperable hepatocellular carcinoma (SARAH): an open-label randomised controlled phase 3 trial. Lancet Oncol 2017;18:1624-36.

11. Llovet JM, Finn RS. Negative phase 3 study of (90)Y microspheres versus sorafenib in HCC. Lancet Oncol 2018;19:e69.

12. Rognoni C, Ciani O, Sommariva S, et al. Trans-arterial radioembolization in intermediate-advanced hepatocellular carcinoma: systematic review and meta-analyses. Oncotarget 2016;7:72343-55.

13. Chiesa C, Maccauro M, Romito R, et al. Need, feasibility and convenience of dosimetric treatment planning in liver selective internal radiation therapy with (90)Y microspheres: the experience of the National Tumor Institute of Milan. Q J Nucl Med Mol Imaging 2011;55:168-97.

14. Spreafico C, Sposito C, Vaiani M, et al. Development of a prognostic score to predict response to Yttrium-90 radioembolization for hepatocellular carcinoma with portal vein invasion. J Hepatol 2018. [Epub ahead of print].

15. Kokudo T, Hasegawa K, Matsuyama Y, et al. Survival benefit of liver resection for hepatocellular carcinoma associated with portal vein invasion. J Hepatol 2016;65:938-43.

Cite this article as: Sposito C, Mazzaferro V. The SIRveNIB and SARAH trials, radioembolization $v s$. sorafenib in advanced HCC patients: reasons for a failure, and perspectives for the future. HepatoBiliary Surg Nutr 2018;7(6):487-489. doi: 10.21037/hbsn.2018.10.06 